\title{
Valley-dependent spin-orbit torques in two-dimensional hexagonal crystals
}

\author{
Hang Li, Xuhui Wang, ${ }^{*}$ and Aurélien Manchon ${ }^{\dagger}$ \\ King Abdullah University of Science and Technology (KAUST), Physical Science and Engineering Division, \\ Thuwal 23955-6900, Saudi Arabia
}

(Received 27 September 2015; published 11 January 2016)

\begin{abstract}
We study spin-orbit torques in two-dimensional hexagonal crystals such as graphene, silicene, germanene, and stanene. The torque possesses two components, a fieldlike term due to inverse spin galvanic effect and an antidamping torque originating from Berry curvature in mixed spin- $k$ space. In the presence of staggered potential and exchange field, the valley degeneracy can be lifted and we obtain a valley-dependent Berry curvature, leading to a tunable antidamping torque by controlling the valley degree of freedom. The valley imbalance can be as high as $100 \%$ by tuning the bias voltage or magnetization angle. These findings open new venues for the development of current-driven spin-orbit torques by structural design.
\end{abstract}

DOI: 10.1103/PhysRevB.93.035417

\section{INTRODUCTION}

Inverse spin galvanic effect (ISGE), referring to the electrical or optical generation of a nonequilibrium spin density in noncentrosymmetric materials, has attracted much attention over the years [1-8]. It originates from the momentum relaxation of carriers in an electrical field and their asymmetric redistribution in subbands that are spin-split by spin-orbit coupling [2]. ISGE was first observed in bulk tellurium and soon generalized to low-dimensional structures such as GaAs quantum wells [3-5].

From an applied perspective, in ferromagnets lacking inversion symmetry, ISGE enables the electrical control of the local magnetization through angular momentum transfer, a mechanism called spin-orbit torque (SOT) $[7,8]$. This effect has been scrutinized in dilute magnetic semiconductors such as ferromagnetic bulk (Ga,Mn)As [9-12] and metallic multilayers comprising heavy metals and ferromagnets [13-18]. These observations have been recently extended to bilayers involving topological insulators displaying extremely large SOT efficiencies $[19,20]$. We note that in metallic multilayers, the spin Hall effect in the adjacent heavy metal also leads to a torque [15] (see discussion in Ref. [21]), which complicates the interpretation of the underlying physics.

From a theoretical perspective, the torque stemming from ISGE on the magnetization $\mathbf{M}$ has the general form

$$
\mathbf{T}=T_{\mathrm{DL}} \mathbf{M} \times(\mathbf{u} \times \mathbf{M})+T_{\mathrm{FL}} \mathbf{M} \times \mathbf{u},
$$

where the first term is called the antidamping-like torque and the second term is referred to as the fieldlike torque [22-27]. The antidamping-like torque is even in the magnetization direction and competes with the magnetic damping, while the fieldlike torque is odd in the magnetization direction and acts like a magnetic field. The vector $\mathbf{u}$ depends on the current direction $\mathbf{j}$ and the symmetries of the spin-orbit coupling. For instance, in a ferromagnetic two-dimensional electron gas (normal to $\mathbf{z}$ ) with Rashba spin-orbit coupling, $\mathbf{u}=\mathbf{z} \times \mathbf{j}$ [7]. An interesting aspect of the formula given above is that the antidamping-like torque arises from the distortion of the wave

\footnotetext{
*xuhuiwangnl@gmail.com

†Aurelien.Manchon@kaust.edu.sa
}

function induced by the electric field, a mechanism closely related to the material's Berry curvature $[12,23,25,27]$.

In parallel to the development of SOT in ferromagnetic structures, the study of spin-orbit coupled transport has also been extended to low-dimensional hexagonal crystals such as graphene. Experimentally, a spin-splitting induced by Rashba spin-orbit coupling has been observed in graphene grown on heavy metals or surface alloys [28-30]. Furthermore, a ferromagnetic insulator $\mathrm{EuO}$ was successfully deposited on graphene and spin-polarized states were detected [31-33]. The recent fabrication of low-dimensional hexagonal crystals with strong intrinsic spin-orbit coupling such as silicene [34,35], germanene [36], and possibly stanene [37], has enriched perspectives in graphene physics. These materials offer a rich platform for the investigation of spin, orbital, and valley-dependent phenomena $[38,39]$.

In this paper, we theoretically investigate the nature of SOT in two-dimensional hexagonal IV group elements crystals such as graphene, silicene, germanene, and stanene. As a matter of fact, the wide tunability of their model band structure presents an appealing opportunity to study the impact of the band geometry (e.g., their Berry curvature) on nonequilibrium mechanisms. Using Kubo formula, we investigate the impact of the band structure on the different components of SOT. We find that intrinsic spin-orbit coupling affects the antidampinglike and fieldlike components differently. The former is sensitive to the presence of a staggered potential while the latter is not. We understand these results in terms of Berry curvature origin of the antidamping torque. The presence of both magnetization and staggered potential enables the emergence of a valley-dependent antidamping torque, providing an additional degree of freedom to the system.

\section{MODEL AND METHOD}

A possible structure to realize valley-dependent SOT is a single-layered hexagonal lattice (such as graphene, silicene, germanene or stanene) sandwiched by a ferromagnetic layer and a nonmagnetic substrate [see Fig. 1(a)]. The ferromagnetic layer may be chosen as $\mathrm{EuO}$ [31] or YIG [40], and induces a weak exchange coupling on the spin-polarized carriers. The underlying nonmagnetic substrate can be either a heavy metal (such as $\mathrm{Pt}, \mathrm{W}$, etc.) or a topological insulator and provides 

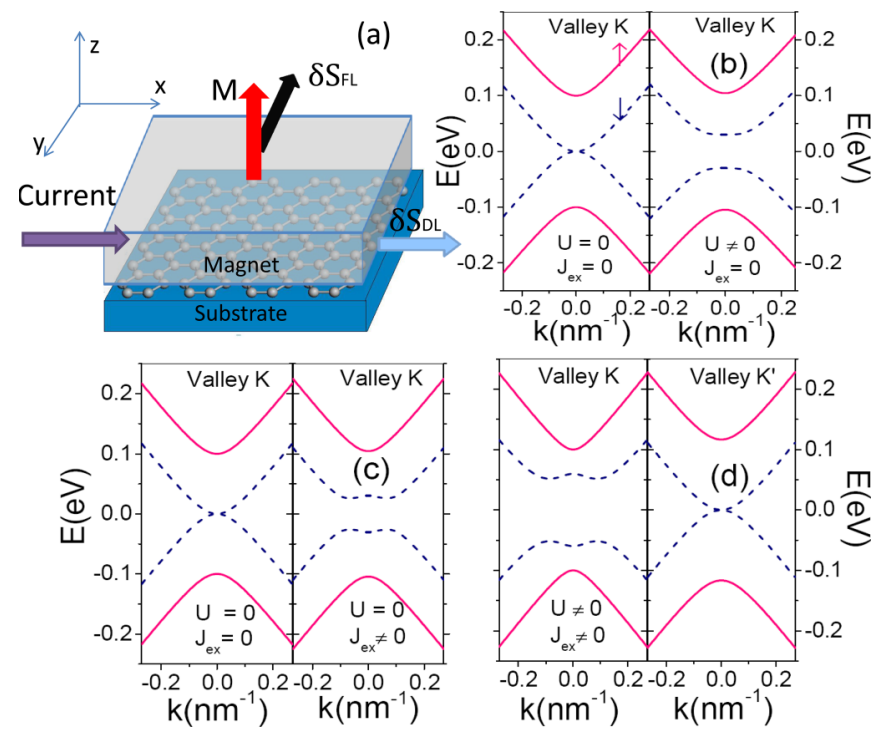

FIG. 1. Schematics of the device based on graphenelike materials with fieldlike and antidamping-like SOT. (b)-(d) Energy dispersion of graphenelike materials with (b) $U=0.03 \mathrm{eV}$ and $J_{\mathrm{ex}}=0$, (c) $U=0$ and $J_{\mathrm{ex}}=0.03 \mathrm{eV}$, and (d) $U=0.03 \mathrm{eV}$ and $J_{\mathrm{ex}}=0.03 \mathrm{eV}$. The pink solid lines and the blue dashed lines denote the spin-up and spin-down subbands, respectively. The current flows from left to right. Magnetization is assumed to be directed along the $z$ axis.

Rashba spin-orbit coupling [29,30,41,42]. Topological insulators present the advantage of having both strong interfacial spin orbit coupling and vanishing bulk conductance [41], thereby promoting carrier transport at their surface. Note that in principle, a magnetic insulator alone could supply for both exchange field and Rashba spin-orbit coupling [20,43,44].

The concept of valley-dependent SOT is illustrated in Fig. 1(a). In the absence of a magnet, the interaction between the substrate and graphenelike layer breaks the inversion symmetry and leads to Rashba spin-orbit coupling. As a result, a transverse nonequilibrium spin density builds up when a current is injected along the horizontal direction. Both Rashba and intrinsic spin-orbit coupling are valley dependent as shown in Eq. (2) and thus they can not break the valley degeneracy. In the presence of a magnet, a fieldlike spin density and an antidamping-like spin density are generated as shown in Fig. 1(a) $[14,15]$. The exchange field only breaks the time-reversal symmetry, while the sublattice symmetry (two-fold rotational symmetry in the plane) is preserved as shown in Fig. 1(c). The interaction between the substrate and graphenelike layer can also induce a staggered potential, which enlarges the band gap without affecting the valley degeneracy, as shown in Fig. 1(b).

However, in the presence of both staggered potential and ferromagnetic exchange field, the valley degeneracy is lifted since both the time-reversal and sublattice symmetries are broken as shown in Fig. 1(d). As a result, SOT becomes valley dependent. Furthermore, as discussed in the next section, the band structure distortion displayed in Figs. 1(b)-1(d) affects the magnitude of the SOT components.

We adopt a low-energy continuum model Hamiltonian which describes Dirac electrons near the Fermi energy and captures the physics behind the formation of the valley-dependent
SOT in the vicinity of $K$ and $K^{\prime}$ points. The total Hamiltonian at $K$ or $K^{\prime}$ valley in the basis of $\left\{\psi_{A, \uparrow}, \psi_{B, \downarrow}, \psi_{B, \uparrow}, \psi_{A, \downarrow}\right\}$ reads [45]

$$
\begin{aligned}
H_{\mathrm{sys}} & =v\left(\tau k_{x} \hat{\sigma}_{x}-k_{y} \hat{\sigma}_{y}\right) \otimes \hat{\mathrm{I}}+\frac{\lambda R}{2}\left(\tau \hat{\sigma}_{x} \otimes \hat{s}_{y}-\hat{\sigma}_{y} \otimes \hat{s}_{x}\right) \\
& +\tau \lambda_{\mathrm{so}} \hat{\sigma}_{z} \otimes \hat{s}_{z}+J_{\mathrm{ex}} \hat{\mathrm{I}} \otimes \mathbf{M} \cdot \hat{\mathbf{s}}+U \hat{\sigma}_{z} \otimes \hat{\mathrm{I}}
\end{aligned}
$$

where $v=\sqrt{3} a t / 2$ with $t$ being a nearest-neighbor hopping parameter, $\tau=+1(-1)$ stands for the $\mathrm{K}$ (or $\mathrm{K}^{\prime}$ ) valley, $\hat{\mathrm{I}}$ is a $2 \times 2$ unity matrix, $a$ is the lattice constant, and $J_{\mathrm{ex}}$ is the ferromagnetic coupling constant. $\hat{\boldsymbol{\sigma}}$ and $\hat{\mathbf{s}}$ are Pauli matrices denoting the AB-sublattice and spin degrees of freedom, respectively. $\mathbf{M}$ is the magnetization direction. The first term includes the spin-independent kinetic energy of the particle, the second term denotes Rashba spin-orbit coupling and the third one represents the intrinsic spin-orbit coupling. The fourth term is the interaction between the spin of the carrier and the local moment of the ferromagnetic system. The last term is the staggered potential (induced, for instance, by an electric field or a substrate [46-48]).

To compute the current-induced effective magnetic field, we first evaluate the nonequilibrium spin density $\delta \mathbf{S}$ at $K\left(K^{\prime}\right)$ valley using Kubo formula [12]:

$$
\begin{aligned}
\delta \mathbf{S}_{K\left(K^{\prime}\right)}= & \frac{e \hbar}{2 \pi V} \operatorname{Re} \sum_{\mathbf{k}, a, b}\left\langle\psi_{\mathbf{k} b}|\hat{\mathbf{s}}| \psi_{\mathbf{k} a}\right\rangle\left\langle\psi_{\mathbf{k} a}|\mathbf{E} \cdot \hat{\mathbf{v}}| \psi_{\mathbf{k} b}\right\rangle \\
& \times\left[G_{\mathbf{k} b}^{R} G_{\mathbf{k} a}^{A}-G_{\mathbf{k} b}^{R} G_{\mathbf{k} a}^{R}\right]
\end{aligned}
$$

where $\mathbf{E}$ is the electric field, $\hat{\mathbf{v}}=\frac{1}{\hbar} \frac{\partial H}{\partial \mathbf{k}}$ is the velocity operator, and $G_{\mathbf{k} a}^{R}=\left(G_{\mathbf{k} a}^{A}\right)^{*}=1 /\left(E_{F}-E_{\mathbf{k} a}+i \Gamma\right) . E_{F}$ is the Fermi energy, $E_{\mathbf{k} a}$ is the energy of electrons in band $a$ and $\Gamma$ is the disorder-induced energy spectral broadening due to the finite lifetime of state $\left|\psi_{\mathbf{k}, a}\right\rangle$ in the presence of impurities. The eigenvector $\left|\psi_{\mathbf{k}, a}\right\rangle$ in band $a$ can be found by diagonalizing Eq. (2).

Equation (3) contains both intraband ( $a=b$ ) and interband $(a \neq b)$ contributions to the nonequilibrium spin density (explicit expressions in the weak $\Gamma$ limit can be found in Ref. [25]). The former stems from the perturbation of the carrier distribution function by the electric field and it is inversely proportional to $\Gamma$, i.e., proportional to the current flow. The latter arises from the distortion of the carrier wave functions by the electric field. This interband contribution also depends on $\Gamma$ but survives when $\Gamma \rightarrow 0$.

In order to evaluate the current-driven SOT in different materials, we define the electrical efficiency of the torque as [7]

$$
\eta=\frac{2 J_{\mathrm{ex}} \delta S}{\hbar \sigma_{x x} \mathrm{E}},
$$

where $\sigma_{i j}$ is the conductivity tensor component defined as [49]

$$
\begin{aligned}
\sigma_{i j} & =e^{2} \hbar \operatorname{Re} \sum_{\mathbf{k}, a, b}\left[\left\langle\psi_{\mathbf{k} a}\left|\hat{\mathbf{v}}_{i}\right| \psi_{\mathbf{k} b}\right\rangle\left\langle\psi_{\mathbf{k} b}\left|\hat{\mathbf{v}}_{j}\right| \psi_{\mathbf{k} a}\right\rangle\right] \\
& \times\left(G_{\mathbf{k} b}^{R} G_{\mathbf{k} a}^{A}-G_{\mathbf{k} b}^{R} G_{\mathbf{k} a}^{R}\right) .
\end{aligned}
$$

At this stage, we wish to point out the limits of the approach outlined above. First, in the present work we consider short-range $(\delta$-like) nonmagnetic impurities. We disregard 
other types of defects such as ripples, magnetic defects [38], or topological dislocations [50], which may have a significant impact on transport in graphene [51]. Second, we limit our calculations to the first Born approximation without calculating the vertex corrections. It is well known that vertex corrections are crucial to model spin-orbit coupled transport in systems with high symmetry such as Rashba two-dimensional electron gas $[52,53]$. However, while these corrections have been found to exactly cancel the intrinsic spin Hall effect [52] and antidamping torque in the presence of spin-independent impurities [53], it seems that they are much less dramatic in graphene, only resulting is a renormalization factor of the order of unity $[54,55]$. Nonetheless, we also notice that recent calculations by Ado et al. [56] indicate that extending the vertex correction beyond the usual noncrossing diagrams (i.e., including $\Psi$ and $X$ crossing diagrams) produces non-negligible impurity-independent contributions. Such contributions could indeed be important for the quantities of interest in this work but are left to further studies.

\section{INVERSE SPIN GALVANIC EFFECT}

The characteristics of the SOT in two-dimensional hexagonal honeycomb lattices are expected to be different from the well studied cases of two-dimensional magnetic Rashba gas $[7,8,22,23]$ or bulk GaMnAs [24,25]. Unlike the threedimensional ferromagnetic GaMnAs in the weak limit $\left(\lambda_{R} \ll\right.$ $J_{\mathrm{ex}}, J_{\mathrm{ex}} \sim 1 \mathrm{eV}$ and $\left.\lambda_{R} \sim 0.1 \mathrm{eV}\right)$, the graphenelike materials often fall into the strong limit $\left(\lambda_{R} \gg J_{\mathrm{ex}}\right)$ leading to sizable interband contributions. The nontrivial Dirac kinetic term [first term in Eq. (2)] gives rise to nonlinear transitions of spin density when tuning the Fermi energy. Furthermore, the spin density is more sensitive to band topology tunable by intrinsic spin-orbit coupling or staggered potential. Most importantly, the Dirac kinetic term and spin-orbit coupling terms are valley-dependent. In order to better understand the valley-dependent SOT, we first examine spin torque with valley degeneracy in the present section. The influence of valley degeneracy lifting will be investigated in Sec. IV.

\section{A. Nonmagnetic honeycomb lattice}

We first compute the spin density induced by ISGE in nonmagnetic graphene. In this paper, we choose the following parameters: $E_{f} \in[0,0.3] \mathrm{eV}$ [57], $\lambda_{R} \in[10,130] \mathrm{meV}$ $[29,30]$, and $J_{\mathrm{ex}} \in[5,30] \mathrm{meV}[58,59]$. For all the calculations shown in this paper, the electric field is assumed to be along $x$ axis and the energy broadening is $\Gamma=0.01 \mathrm{eV}$. To understand the physical origin of the SOT and establish connections with previous works (such as Ref. [25]), we parse the SOT into intraband and interband contributions.

Figure 2 presents the intraband [(a) and (c)] and interband contributions [(b) and (d)] to the ISGE-driven spin density for various strengths of $\lambda_{R}$ [(a) and (b)] and $\lambda_{\text {so }}$ [(c) and (d)]. In nonmagnetic graphene, the intraband contribution produces a spin density aligned toward the $y$ direction, which is expected from the geometry of our system and consistent with the well known ISGE in two-dimensional electron gases [7,60]. There is also a nonignorable interband contribution in the strong limit $\left(\lambda_{R} \gg J_{\mathrm{ex}}\right)$, smaller than the intraband contribution and opposite to it, in agreement with our previous analytical

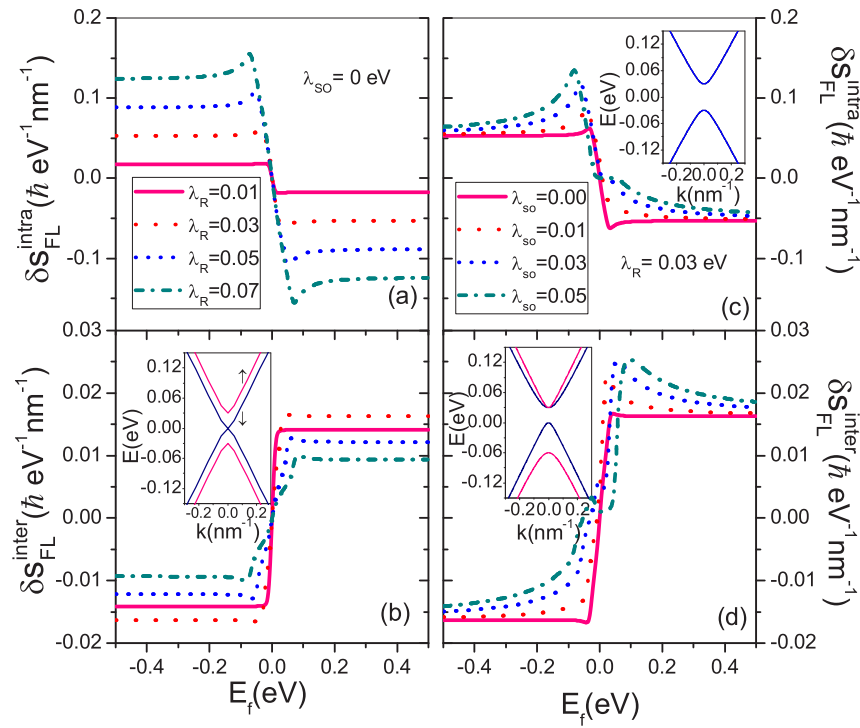

FIG. 2. (a) Intraband and (b) interband contributions to spin density as a function of Fermi energy $E_{f}$ for various Rashba spin-orbit coupling in the absence of intrinsic spin-orbit coupling $\lambda_{\text {so }}$. (c) Intraband and (d) interband spin density as a function of Fermi energy $E_{f}$ for various intrinsic spin-orbit coupling at $\lambda_{R}=0.03 \mathrm{eV}$. Inset (b) Band structure of graphenelike materials with $\lambda_{R}=0.03 \mathrm{eV}$ and $\lambda_{\text {so }}=0 \mathrm{eV}$. Inset (c) Band structure with $\lambda_{R}=0 \mathrm{eV}$ and $\lambda_{\text {so }}=0.03$ $\mathrm{eV}$. Inset (d) Same as inset (b) but with $\lambda_{\mathrm{so}}=0.03 \mathrm{eV}$. The current is injected along the $x$ axis.

solutions in the case of Rashba two-dimensional electron gas [25]. When increasing the absolute value of Fermi energy, the spin density first experiences a sharp enhancement at small values of $E_{f}$ and quickly saturates. This result is consistent with Ref. [61] and can be readily understood by considering the band structure in the inset of Fig. 2(b). When the Fermi energy lies in the energy gap of two spin-split subbands, only one spin species contributes to ISGE and the intraband spin density increases with the Fermi energy. As the Fermi energy lies above the subband gap, the two subbands compensate each other and the spin density saturates. The peaks in Fig. 2(a) correspond to the minimum $\left(E_{f}>0\right)$ or maximum $\left(E_{f}<0\right)$ of the spin-up subband [see inset of Fig. 2(b)], which is of the order of $\lambda_{R}$.

Another interesting feature is the spin density as a function of Rashba spin-orbit coupling. The intraband contribution increases linearly with $\lambda_{R}$ [see Fig. 2(a)], while the interband contribution first increases and then decreases [see Fig. 2(b)]. The interband contribution depends on the energy difference between the subbands, which itself is of the order of $\lambda_{R}$. Indeed, one can show that in the weak-impurity limit, the interband contribution is proportional to $1 /\left(E_{\mathbf{k} a}-E_{\mathbf{k} b}\right)[12,25,27]$. This results in the nonlinear dependence as a function of $\lambda_{R}$ observed in Fig. 2(b) as well as in Fig. 3(c).

Rashba spin-orbit coupling is not the only spin-orbit coupling that affects the spin density. In graphenelike systems, Rashba spin-orbit coupling is always accompanied by an intrinsic spin-orbit coupling, $\sim \tau \lambda_{\mathrm{so}} \hat{\sigma}_{z} \otimes \hat{s}_{z}$, which originates from the substrate or a low buckled structure [46,62]. In Figs. 2(c) and 2(d), we display the Fermi energy dependence of the intraband and interband contributions to spin density for 


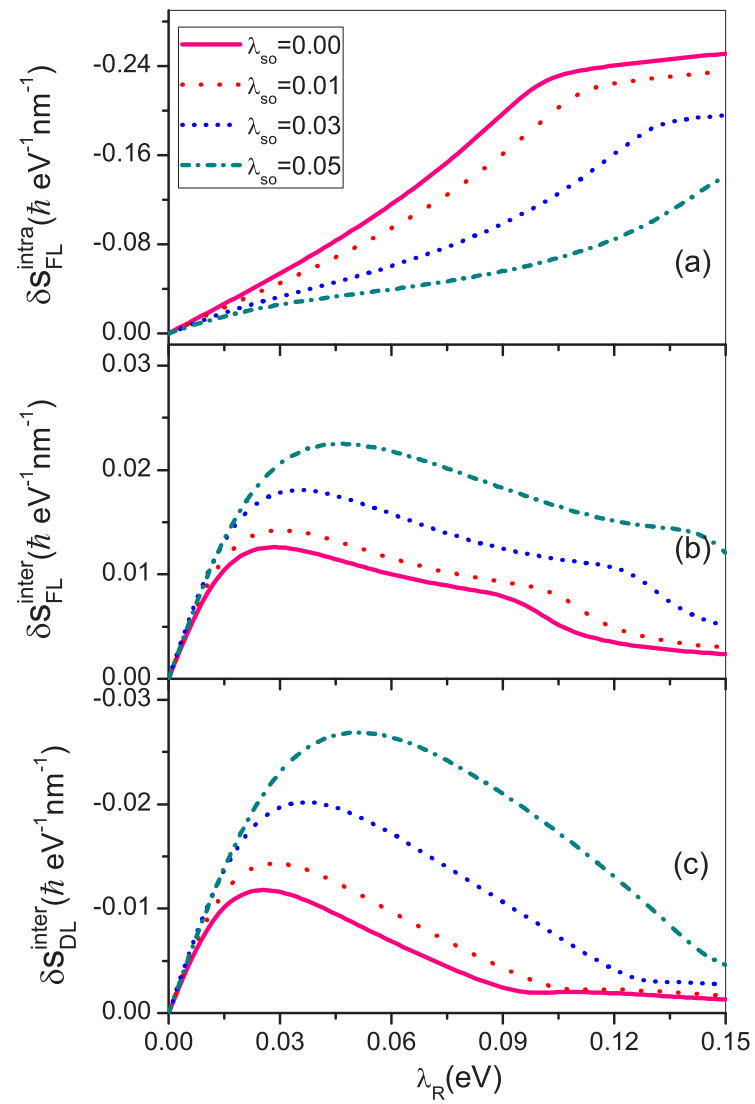

FIG. 3. (a) Intraband and (b)-(c) interband spin density as a function of Rashba spin-orbit coupling for different intrinsic spin-orbit coupling with $J_{\mathrm{ex}}=0.01 \mathrm{eV}$ and $E_{f}=0.1 \mathrm{eV}$. The magnetization is directed along the $z$ axis.

various intrinsic spin-orbit coupling strengths. As expected, the intrinsic spin-orbit coupling opens up a band gap and distorts the topology of the band structure as seen in the inset of Figs. 2(c) and 2(d). For a given $K$ or $K^{\prime}$ valley $(\tau= \pm 1)$, this term changes sign between the two sublattices $\left(\sim \hat{\sigma}_{z}\right)$, playing the same role as an antiferromagnetic exchange field along $z$ axis. As a result, the profile of the spin density is asymmetric between the two sublattices, as reflected by a shift towards negative energies $\left(E_{f}<0\right)$, see Fig. 2(c). The asymmetry of the profiles of the spin density becomes more evident with the increase of $\lambda_{\mathrm{so}}$. The energy at which the spin density is maximum equals $-\left|\lambda_{\mathrm{so}}+\lambda_{R}\right|$ when $E_{f}<0$. Note that the intrinsic spin-orbit coupling does not drive ISGE by itself, but it affects the ISGE-induced spin density driven by Rashba spin-orbit coupling through the modulation of the topology of the bands.

\section{B. Magnetic honeycomb lattice}

Let us now turn to the case of magnetic two-dimensional honeycomb lattices. To understand the role of spin-orbit coupling, we plot the intraband and interband spin densities as a function of Rashba spin-orbit coupling for different intrinsic spin-orbit coupling in the presence of magnetization in Fig. 3. Due to the presence of magnetism, the interband contribution also produces an antidamping component [see Fig. 3(c)], i.e., a spin density contribution oriented towards $\sim \mathbf{M} \times \mathbf{y}[12,25,27]$ and with a magnitude comparable to the one of the fieldlike component [see Fig. 3(c)]. As seen in Figs. 3(a)-3(c) the interband fieldlike and antidamping contributions first increase and then decrease. This can be understood as a competition between the spin density driven by Rashba spin-orbit coupling and the suppression of interband scattering due to the distance between the subbands that increases with $\lambda_{R}$.

The intraband contribution decreases with the increasing intrinsic spin-orbit coupling while the interband contribution behaves the opposite way. By opening a band gap, the intrinsic spin-orbit coupling alters the band filling, resulting in a reduced intraband contribution to spin density. An analytical solution of energy depending on intrinsic spin-orbit coupling can be found in Ref. [63]. On the other hand, the intrinsic spin-orbit coupling reduces the splitting between the subbands for $E_{f}>0$ [see inset in Fig. 2(d)], which results in an enhancement of the interband contributions. This result is valuable to currentdriven magnetic excitations since the antidamping torque is responsible for magnetization switching and excitations [14,15] (see, for instance, also Ref. [64]).

\section{VALLEY-DEPENDENT SPIN-ORBIT TORQUE}

The valley degree of freedom can be used as a tool to enhance the functionality of two-dimensional honeycomb lattices [65]. Recently, a valley-dependent anomalous quantum Hall state has been predicted in silicene and silicene nanoribbons associated with a topological phase transition [46,66,67], and a charge-neutral Hall effect has been measured in graphene devices $[68,69]$. The latter observations suggest the emergence of valley Hall effect. It is thus natural to expect a valley-modulated SOT in our system.

\section{A. Staggered potential}

The sublattice degeneracy can be removed by depositing graphenelike materials on hexagonal boron nitride $[68,70,71]$ or silicon carbide [48], or by applying an electric field in a low buckled structure [46]. When the staggered potential and exchange field are present and the valley degeneracy is broken, the spin density becomes valley-dependent as shown in Fig. 4.

In Figs. 4(a)-4(c), we display the intraband and interband contributions to spin density as a function of Fermi energy in the presence of staggered potential with and without the intrinsic spin-orbit coupling. The imbalance between the contribution of the two valleys to the spin density, i.e., valley polarization, defined as $P=\frac{\delta \mathbf{S}_{K}-\delta \mathbf{S}_{K^{\prime}}}{\delta \mathbf{S}_{K}+\delta \mathbf{S}_{K^{\prime}}}$, is reported on Figs. 4(d) $-4(f)$. The largest imbalance occurs mainly around the neutrality point $E_{f}=0$. The valley imbalance of the antidampinglike component can reach $100 \%$ as shown in Fig. 4(f), i.e., for certain energies this component is dominated by only one valley. When the intrinsic spin-orbit coupling is present, the magnitudes of the valley imbalance can be switched from $-100 \%$ to $100 \%$ by simply tuning the Fermi energy.

\section{B. Angular dependence}

A noticeable effect of lifting the valley degeneracy is its impact on the angular dependence of SOT components. Figure 5 displays the angular dependence of the different components of the spin density when the magnetization is rotated in the $(x, z)$ plane. In a ferromagnetic two-dimensional electrons gas 


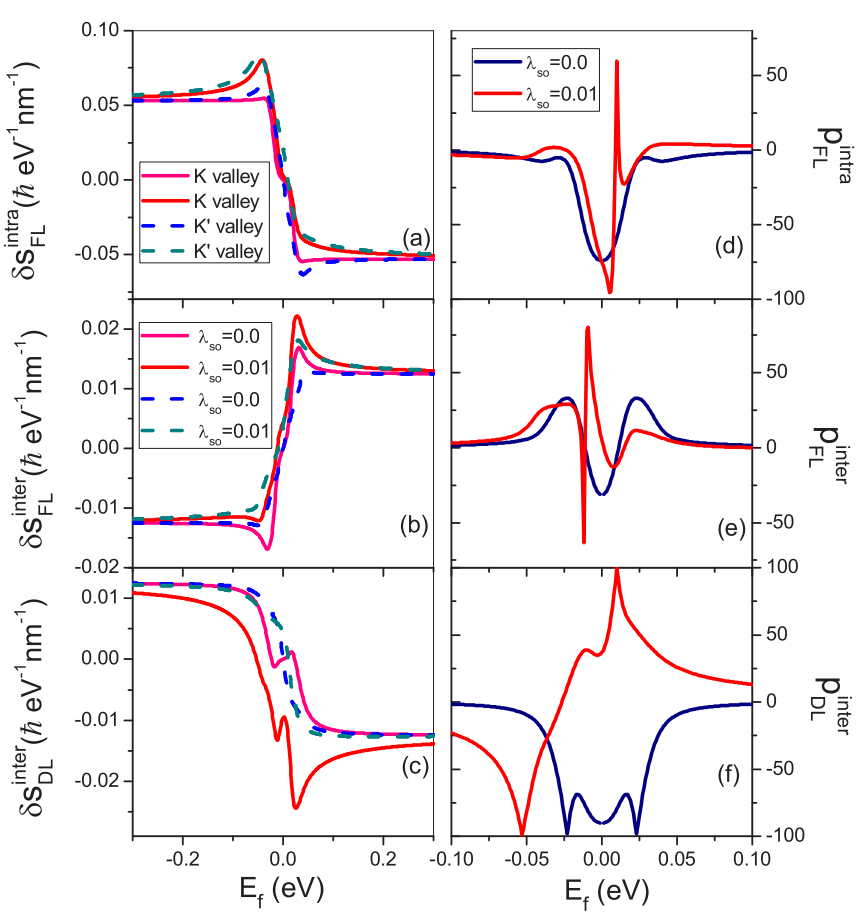

FIG. 4. (a) Intraband and (b)-(c) interband spin density of two valleys as a function of Fermi energy for different intrinsic spin-orbit coupling with $U=0.01 \mathrm{eV}$ and $J_{\mathrm{ex}}=0.01 \mathrm{eV}$. Valley polarization for intraband (d) and interband (e)-(f) components for different intrinsic spin-orbit coupling.

with Rashba spin-orbit coupling, the spin density has the general form $\delta \mathbf{S}=\delta S_{\|} \cos \theta \mathbf{x}+\delta S_{y} \mathbf{m} \times \mathbf{y}-\sin \theta \delta S_{\|} \mathbf{z}$ (e.g., see Ref. [25]), where $\theta$ is the angle between the magnetization and $\mathbf{z}$. A more complex angular dependence may appear in the strong Rashba limit $\left(\lambda_{R} \gg J_{\mathrm{ex}}\right)$ due to D'yakonov-Perel relaxation [72] or in the intermediate regime $\left(\lambda_{R} \sim J_{\mathrm{ex}}\right)$ due to "breathing" Fermi surface [27] (see below).

Similarly, in the case of magnetic honeycomb lattices, the different components of the spin density display a clear deviation from the simple $\sim \cos \theta$ dependence of the ferromagnetic

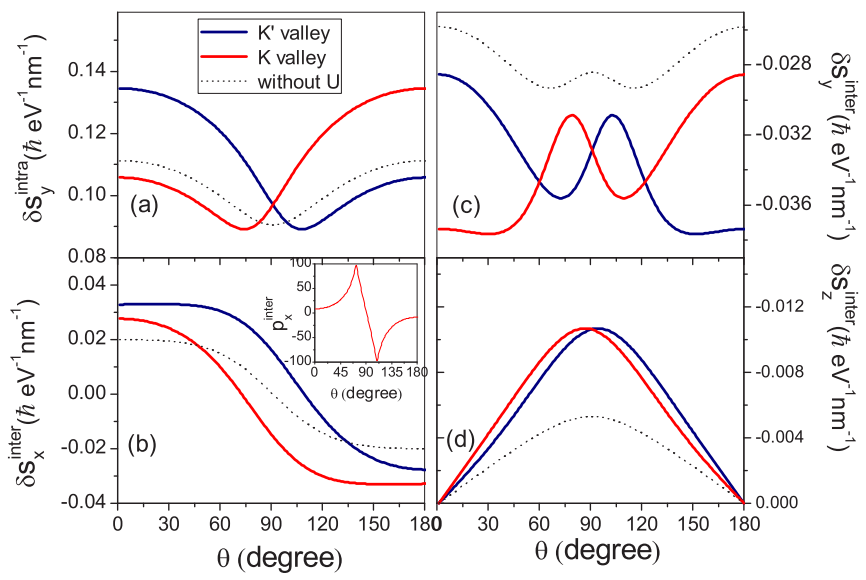

FIG. 5. Intraband and interband spin density as a function of the magnetization direction with (solid lines) and without (dashed lines) staggered potential for the different valleys when $U=0.03 \mathrm{eV}$. Inset (b) Valley polarization of interband spin density for $x$ component.
Rashba gas (see dotted lines in Fig. 5). This is attributed to the "breathing" Fermi surface, i.e., the distortion of the Fermi surface, and the modification of the band filling as a function of the direction of the magnetization when the exchange is comparable to the Rashba parameter.

In the absence of valley degeneracy, the angular dependencies at $K$ and $K^{\prime}$ points differ significantly from each other (red and blue lines in Fig. 5, respectively). As a consequence, by tuning the magnetization angle the valley imbalance varies strongly [from $-100 \%$ to $100 \%$ for $x$-component, as shown in inset of in Fig. 5(b)]. We also notice that additional structures are visible in the angular dependence of the fieldlike component, related to the interband transitions [see Fig. 5(c)]. These features are unique to the case of honeycomb lattices and absent in standard two-dimensional free electron gases.

\section{CONNECTION BETWEEN SPIN-ORBIT TORQUE AND BERRY CURVATURE}

Berry's phase plays a crucial role in the transport properties of semiconductors especially for graphenelike materials. Due to the inequivalent contribution from the two valleys, Berry curvature induces a valley Hall effect in graphene with broken inversion symmetry [68]. Recently, the link between SOT and Berry curvature was established in bulk ferromagnetic GaMnAs [12].

In order to show the connection between the SOT and the band structure distortion, let us analyze the influence of intrinsic spin-orbit coupling on SOT in the presence of a staggered potential. The intraband and interband contributions to spin density as a function of intrinsic spin-orbit coupling

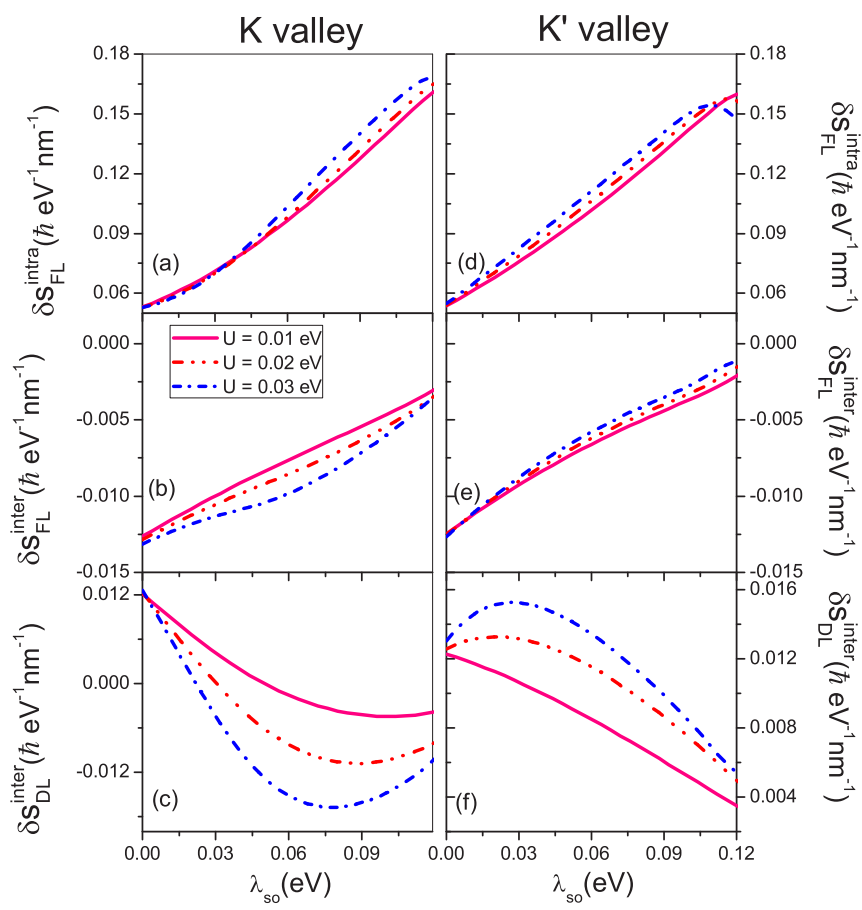

FIG. 6. Intraband and interband spin density as a function of intrinsic spin-orbit coupling for a different staggered potential for the $K$ valley (a)-(c) and $K^{\prime}$ valley (d)-(f). The parameters are $E_{f}=$ $-0.16 \mathrm{eV}, J_{\mathrm{ex}}=0.01 \mathrm{eV}$, and $\lambda_{R}=0.03 \mathrm{eV}$. 


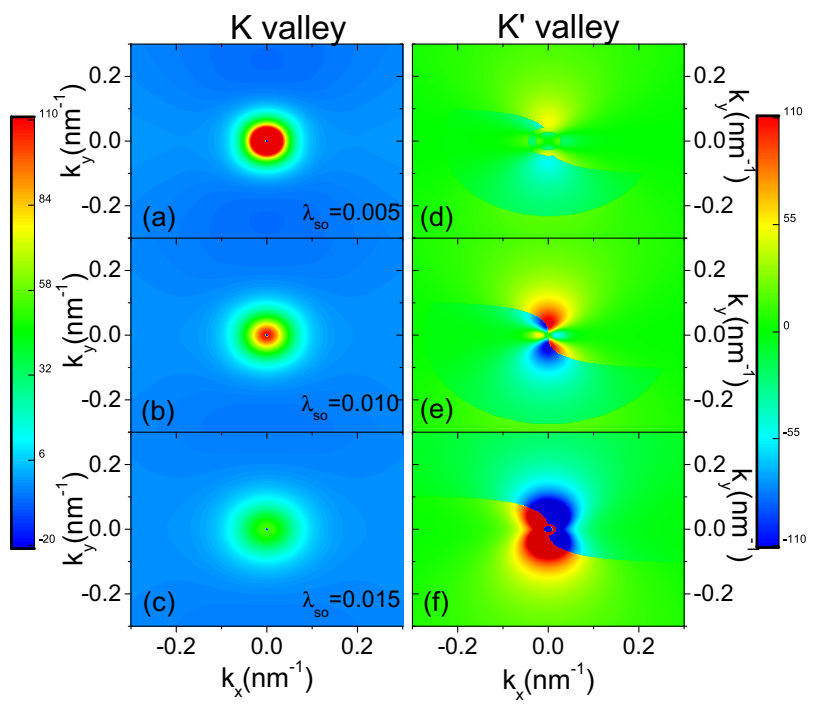

FIG. 7. Contour of valley-polarized Berry curvature distribution for different intrinsic spin-orbit coupling in $k_{x}-k_{y}$ plane with $U=$ $0.03 \mathrm{eV}$. [(a) and (d)] $\lambda_{\text {so }}=0.005 \mathrm{eV}$. [(b) and (e)] $\lambda_{\text {so }}=0.01 \mathrm{eV}$. [(c) and (f) $] \lambda_{\text {so }}=0.015 \mathrm{eV}$. Others parameters are the same as in Fig. 6.

for various staggered potentials both at $K$ and $K^{\prime}$ valleys are displayed in Fig. 6. We find that both the fieldlike intraband and interband contributions to the spin density, $\delta S_{\mathrm{FL}}^{\text {intra }}$ and $\delta S_{\mathrm{FL}}^{\text {inter }}$, increase with the intrinsic spin-orbit coupling and are only weakly affected by the staggered potential [see Figs. 6(a), 6(b), 6(d), and 6(e)]. In contrast, the antidampinglike component of the spin density, $\delta S_{\mathrm{DL}}^{\text {inter }}$, displays a nonlinear dependence as a function of the intrinsic spin-orbit coupling that is very different for the two valleys and highly sensitive to the staggered potential [see Figs. 6(c) and 6(f)].

To understand this difference, we plot the contour of Berry curvature for different intrinsic spin-orbit coupling at $K$ and $K^{\prime}$ valleys in $k_{x}-k_{y}$ plane in Fig. 7. A large Berry curvature mainly concentrates around the Dirac point and decays away from it, in agreement with previous results [45,73]. For the $K$ valley, the Berry curvature decreases with the increase of intrinsic spin-orbit coupling. Yet for the $K^{\prime}$ valley, the Berry curvature increases. This trend is in accordance with the variations of $\delta S_{\mathrm{DL}}^{\text {inter }}$ displayed in Figs. 6(c) and 6(f) and contrasts with the variations of $\delta S_{\mathrm{FL}}^{\text {inter }}$ displayed in Figs. 6(b) and 6(e). It illustrates the fact that while $\delta S_{\mathrm{FL}}^{\text {inter }}$ and $\delta S_{\mathrm{DL}}^{\text {inter }}$ both originate from interband transitions, only the latter is related to the Berry curvature.

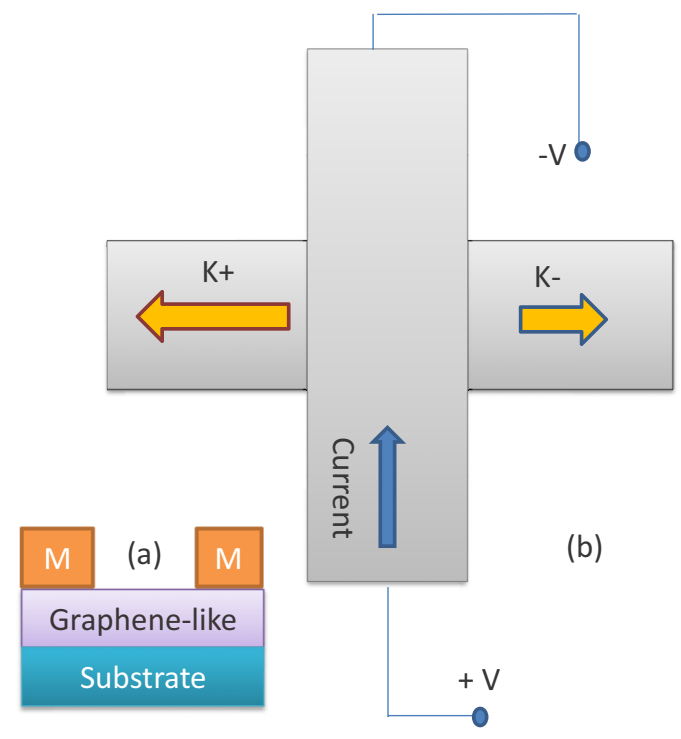

FIG. 8. Schematics of the realization of valley-dependent antidampinglike SOT: (a) top view and (b) side view. The current is injected into the vertical arm. The presence of both magnetization and staggered potential results in a nonequivalent spin density for the valleys. This leads to a different valleys on the horizontal sidearms.

\section{DISCUSSION}

To complete the present study, we computed the magnitude of antidampinglike and fieldlike components of the spin density and corresponding electrical efficiencies for various graphenelike honeycomb lattices, assuming $\lambda_{R}=0.1 \mathrm{eV}$ and $J_{\mathrm{ex}}=0.03 \mathrm{eV}$. The results are reported in Table I, showing that the largest SOT is obtained for stanene $\left[\sim 100 \times 10^{10}\right.$ $\mathrm{eV} /(\mathrm{A} \mathrm{m})]$. As a comparison, the corresponding efficiencies of fieldlike SOT in (Ga,Mn)As [25], two-dimensional Rashba systems [7] and topological insulators [74] are of the order of $\sim 1 \times 10^{10}, \sim 10 \times 10^{10}$, and $\sim 100 \times 10^{10} \mathrm{eV} /(\mathrm{A} \mathrm{m})$, respectively, in agreement with the orders of magnitude reported experimentally $[9,11,14,20]$. Therefore, for moderate Rashba and exchange parameters, honeycomb lattices seem to display large fieldlike torques. Interestingly, the antidampinglike torque remains about one order of magnitude smaller than the fieldlike torque, as already observed in two-dimensional Rashba gases and (Ga,Mn)As [25].

Finally, we propose a device to detect the valley-dependent SOT. We consider a multi-terminal device as shown in Fig. 8. This is a typical device used to detect charge neutral-currents $[68,69]$. The device consists of a graphenelike material

TABLE I. Efficiency of spin torque for various two-dimensional hexagonal lattices.

\begin{tabular}{|c|c|c|c|c|c|c|c|}
\hline & $E(\mathrm{eV})$ & $a(\AA)$ & $\sigma_{x x}\left(e^{2} / \hbar\right)$ & $s_{\mathrm{DL}}\left[\hbar(\mathrm{eV} \mathrm{nm})^{-1}\right]$ & $s_{\mathrm{FL}}\left[\hbar(\mathrm{eV} \mathrm{nm})^{-1}\right]$ & $\eta_{\mathrm{DL}}\left[\mathrm{eV}(\mathrm{A} \mathrm{m})^{-1}\right]$ & $\eta_{\mathrm{FL}}\left[\mathrm{eV}(\mathrm{A} \mathrm{m})^{-1}\right]$ \\
\hline Carb & $2.7^{1}$ & $2.46^{2}$ & $23.3809 \times 10^{-3}$ & 0.0083 & 0.1193 & $2.13 \times 10^{10}$ & $30.6 \times 10^{10}$ \\
\hline Silicene & $1.04^{3}$ & $3.87^{2}$ & $9.0068 \times 10^{-3}$ & 0.0 & 0.1975 & $9.13 \times 10^{10}$ & $131.6 \times 10^{10}$ \\
\hline Germanene & $0.97^{3}$ & $4.06^{2}$ & $8.4004 \times 10^{-3}$ & 0.0141 & 0.2019 & $10.07 \times 10^{10}$ & $144.2 \times 10^{10}$ \\
\hline Stanene & $0.76^{3}$ & $4.67^{2}$ & $6.5818 \times 10^{-3}$ & 0.0155 & 0.220 & $14.13 \times 10^{10}$ & $200.6 \times 10^{10}$ \\
\hline
\end{tabular}

\footnotetext{
${ }^{1}$ Reference [45].

${ }^{2}$ Reference [62].

${ }^{3}$ Reference [75].
} 
sandwiched between a magnetic insulator and a nonmagnetic substrate with strong spin-orbit coupling (such as a topological insulator [41]). The substrate [76] can induce a staggered potential that breaks the valley degeneracy. The voltage is applied to the sidearms and the current flows from the lower sidearm to the upper one. In the absence of magnetization, a valley Hall effect may be detected in the two horizontal terminals [68]. In the presence of magnetization, the torque exerted on the magnetization of the magnetic insulator deposited on top of the left or right terminal will be different.

\section{CONCLUSION}

In summary, we have investigated the nature of SOTs in two-dimensional hexagonal crystals and qualitatively recovered most of the results obtained on different systems such as (Ga,Mn)As and two-dimensional Rashba gases [25]. We showed that the staggered potential and intrinsic spin-orbit coupling can strongly affect the magnitude of the torque components as well as their angular dependence. In the presence of staggered potential and exchange field, the valley degeneracy can be lifted and we obtain a valley-dependent antidamping SOT, while the fieldlike component remains mostly unaffected. This feature is understood in terms of Berry curvature and we show that the valley imbalance can be as high as $100 \%$ by tuning the bias voltage or magnetization angle.

\section{ACKNOWLEDGMENT}

H.L. and A.M. were supported by the King Abdullah University of Science and Technology (KAUST).
[1] E. L. Ivchenko and G. E. Pikus, Pis'ma Zh. Eksp. Theor. Fiz. 27, 640 (1978) [JETP Lett. 27, 604 (1978)]; A. G. Aronov and Yu. B. Lyanda-Geller, Pis'ma Zh. Eksp. Theor. Fiz. 50, 398 (1989) [JETP Lett. 50, 431 (1989)].

[2] E. L. Ivchenko and S. Ganichev, in Spin Physics in Semiconductors, edited by M. I. Dyakonov (Springer, New York, 2008).

[3] L. E. Vorobev, E. L. Ivchenko, G. E. Pikus, I. I. Farbstein, V. A. Shalygin, and A. V. Sturbin, Pis'ma Zh. Eksp. Theor. Fiz. 29, 485 (1979) [JETP Lett. 29, 441 (1979)].

[4] A. Yu. Silov, P. A. Blajnov, J. H. Wolter, R. Hey, K. H. Ploog, and N. S. Averkiev, Appl. Phys. Lett. 85, 5929 (2004).

[5] S. D. Ganichev, S. N. Danilov, P. Schneider, V. V. Belkov, L. E. Golub, W. Wegscheider, D. Weiss, and W. Prettl, J. Magn. Magn. Mater. 300, 127 (2006).

[6] C. Gorini, P. Schwab, M. Dzierzawa, and R. Raimondi, Phys. Rev. B 78, 125327 (2008).

[7] A. Manchon and S. Zhang, Phys. Rev. B 78, 212405 (2008); 79, 094422 (2009).

[8] I. Garate and A. H. MacDonald, Phys. Rev. B 80, 134403 (2009); A. Matos-Abiague and R. L. Rodriguez-Suarez, ibid. 80, 094424 (2009).

[9] A. Chernyshov, M. Overby, X. Liu, J. K. Furdyna, Y. LyandaGeller, and L. P. Rokhinson, Nat. Phys. 5, 656 (2009).

[10] M. Endo, F. Matsukura, and H. Ohno, Appl. Phys. Lett. 97, 222501 (2010).

[11] D. Fang, H. Kurebayashi, J. Wunderlich, K. Výborný, L. P. Zârbo, R. P. Campion, A. Casiraghi, B. L. Gallagher, T. Jungwirth, and A. J. Ferguson, Nat. Nanotechnol. 6, 413 (2011).

[12] H. Kurebayashi, J. Sinova, D. Fang, A. C. Irvine, J. Wunderlich, V. Novak, R. P. Campion, B. L. Gallagher, E. K. Vehstedt, L. P. Zârbo, K. Vyborny, A. J. Ferguson, and T. Jungwirth, Nat. Nanotechnol. 9, 211 (2014).

[13] K. Garello, I. M. Miron, C. O. Avci, F. Freimuth, Y. Mokrousov, S. Blügel, S. Auffret, O. Boulle, G. Gaudin, and P. Gambardella, Nat. Nanotechnol. 8, 587 (2013).

[14] I. M. Miron, G. Gaudin, S. Auffret, B. Rodmacq, A. Schuhl, S. Pizzini, J. Vogel, and P. Gambardella, Nat. Mater. 9, 230 (2010); U. H. Pi, K. W. Kim, J. Y. Bae, S. C. Lee, Y. J. Cho, K. S. Kim, and S. Seo, Appl. Phys. Lett. 97, 162507 (2010); T. Suzuki, S. Fukami, N. Ishiwata, M. Yamanouchi, S. Ikeda, N. Kasai, and
H. Ohno, ibid. 98, 142505 (2011); I. M. Miron et al., Nature (London) 476, 189 (2011).

[15] L. Liu, O. J. Lee, T. J. Gudmundsen, D. C. Ralph, and R. A. Buhrman, Phys. Rev. Lett. 109, 096602 (2012); L. Liu, C.-F. Pai, Y. Li, H. W. Tseng, D. C. Ralph, and R. A. Buhrman, Science 336, 555 (2012).

[16] J. Kim, J. Sinha, M. Hayashi, M. Yamanouchi, S. Fukami, T. Suzuki, S. Mitani, and H. Ohno, Nat. Mater. 12, 240 (2013).

[17] X. Fan, J. Wu, Y. Chen, M. J. Jerry, H. Zhang, and J. Q. Xiao, Nat. Commun. 4, 1799 (2013).

[18] M. Jamali, K. Narayanapillai, X. Qiu, L. M. Loong, A. Manchon, and H. Yang, Phys. Rev. Lett. 111, 246602 (2013).

[19] A. R. Mellnik, J. S. Lee, A. Richardella, J. L. Grab, P. J. Mintun, M. H. Fischer, A. Vaezi, A. Manchon, E. A. Kim, N. Samarth, and D. C. Ralph, Nature (London) 511, 449 (2014).

[20] Y. Fan, P. Upadhyaya, X. Kou, M. Lang, S. Takei, Z. Wang, J. Tang, L. He, L. T. Chang, M. Montazeri, G. Yu, W. Jiang, T. Nie, R. N. Schwartz, Y. Tserkovnyak, and K. L. Wang, Nat. Mater. 13, 699 (2014).

[21] A. Brataas, A. D. Kent, and H. Ohno, Nat. Mater. 11, 372 (2012).

[22] D. A. Pesin and A. H. MacDonald, Phys. Rev. B 86, 014416 (2012).

[23] E. van der Bijl and R. A. Duine, Phys. Rev. B 86, 094406 (2012).

[24] H. Li, X. Wang, F. Dogan, and A. Manchon, Appl. Phys. Lett. 102, 192411 (2013).

[25] H. Li, H. Gao, L. P. Zarbo, K. V. Vyborny, X. Wang, I. Garate, F. Dogan, A. Cejchan, J. Sinova, T. Jungwirth, and A. Manchon, Phys. Rev. B 91, 134402 (2015).

[26] X. Wang and A. Manchon, Phys. Rev. Lett. 108, 117201 (2012).

[27] K. S. Lee, D. Go, A. Manchon, P. M. Haney, M. D. Stiles, H. W. Lee, and K. J. Lee, Phys. Rev. B 91, 144401 (2015).

[28] A. Varykhalov, J. Sanchez-Barriga, A. M. Shikin, C. Biswas, E. Vescovo, A. Rybkin, D. Marchenko, and O. Rader, Phys. Rev. Lett. 101, 157601 (2008).

[29] D. Marchenko, A. Varykhalov, M. Scholz, G. Bihlmayer, E. Rashba, A. Rybkin, A. Shikin, and O. Rader, Nat. Commun. 3, 1232 (2012).

[30] P. Leicht, J. Tesch, S. Bouvron, F. Blumenschein, P. Erler, L. Gragnaniello, and M. Fonin, Phys. Rev. B 90, 241406(R) (2014). 
[31] A. G. Swartz, P. M. Odenthal, Y. Hao, R. S. Ruoff, and R. K. Kawakami, ACS Nano 6, 10063 (2012).

[32] J. Klinkhammer, and M. Schlipf, and F. Craes, and S. Runte, and T. Michely, and C. Busse, Phys. Rev. Lett. 112, 016803 (2014).

[33] J. Klinkhammer, D. Forster, S. Schumacher, Hans P. Oepen, T. Michely, and C. Busse, Appl. Phys. Lett. 103, 131601 (2013).

[34] A. Fleurence, R. Friedlein, T. Ozaki, H. Kawai, Y. Wang, and Y. Yamada-Takamura, Phys. Rev. Lett. 108, 245501 (2012).

[35] P. Vogt, P. De Padova, C. Quaresima, J. Avila, E. Frantzeskakis, M. C. Asensio, A. Resta, B. Ealet, and G. Le Lay, Phys. Rev. Lett. 108, 155501 (2012).

[36] L. Li, S. Lu, J. Pan, Z. Qin, Y. Wang, Y. Wang, G. Y. Cao, S. Du, and H. Gao, Adv. Mater. 26, 4820 (2014).

[37] Y. Xu, B. Yan, H. J. Zhang, J. Wang, G. Xu, P. Tang, W. Duan, and S. C. Zhang, Phys. Rev. Lett. 111, 136804 (2013).

[38] W. Han, R. K. Kawakami, M. Gmitra, and J. Fabian, Nat. Nanotechnol. 9, 794 (2014).

[39] A. Manchon, H. C. Koo, J. Nitta, S. M. Frolov, and R. A. Duine, Nat. Mater. 14, 871 (2015).

[40] Z. Wang, C. Tang, R. Sachs, Y. Barlas, and J. Shi, Phys. Rev. Lett. 114, 016603 (2015).

[41] K. H. Jin and S. H. Jhi, Phys. Rev. B 87, 075442 (2013).

[42] C. Chang et al., Science 340, 167 (2013).

[43] Y. Zou and Y. Wang, Nanoscale 3, 2615 (2011).

[44] Z. Qiao, W. Ren, H. Chen, L. Bellaiche, Z. Zhang, A. H. MacDonald, and Q. Niu, Phys. Rev. Lett. 112, 116404 (2014).

[45] Z. Qiao, S. A. Yang, W. Feng, W. K. Tse, J. Ding, Y. Yao, J. Wang, and Q. Niu, Phys. Rev. B 82, 161414(R) (2010).

[46] M. Ezawa, Phys. Rev. Lett. 109, 055502 (2012); see also M. Tahir, A. Manchon, K. Sabeeh, and U. Schwingenschlögl, Appl. Phys. Lett. 102, 162412 (2013).

[47] C. L. Kane and E. J. Mele, Phys. Rev. Lett. 95, 146802 (2005); G. Li, A. Luican, D. Abanin, L. Levitov, and E. Y. Andrei, Nat. Commun. 4, 1744 (2013).

[48] S. Y. Zhou, G. H. Gweon, A. V. Fedorov, P. N. First, W. A. de Heer, D. H. Lee, F. Guinea, A. H. Castro Neto, and A. Lanzara, Nat. Mater. 6, 770 (2007).

[49] Y. Murayama, Mesoscopic Systems: Fundamentals and Applications (Wiley-VCH, Weinheim, Germany, 2001).

[50] Oleg V. Yazyev and Yong P. Chen, Nat. Nanotechnol. 9, 755 (2014).

[51] F. Gargiulo and O. V. Yazyev, Nano Lett. 14, 250 (2014).

[52] J. I. Inoue, G. E. W. Bauer, and L. W. Molenkamp, Phys. Rev. B 70, 041303(R) (2004).

[53] A. Qaiumzadeh, R. A. Duine, and M. Titov, Phys. Rev. B 92, 014402 (2015).

[54] A. Dyrdal and J. Barnas, Phys. Rev. B 92, 165404 (2015).
[55] N. A. Sinitsyn, J. E. Hill, Hongki Min, Jairo Sinova, and A. H. MacDonald, Phys. Rev. Lett. 97, 106804 (2006).

[56] I. A. Ado, I. A. Dmitriev, P. M. Ostrovsky, and M. Titov, Europhys. Lett. 111, 37004 (2015).

[57] D. Pesin and A. H. MacDonald, Nat. Mater. 11, 409 (2012).

[58] H. Haugen, D. Huertas-Hernando, and A. Brataas, Phys. Rev. B 77, 115406 (2008).

[59] H. X. Yang, A. Hallal, D. Terrade, X. Waintal, S. Roche, and M. Chshiev, Phys. Rev. Lett. 110, 046603 (2013).

[60] V. M. Edelstein, Solid State Commun. 73, 233 (1990).

[61] A. Dyrdal, J. Barnas, and V. K. Dugaev, Phys. Rev. B 89, 075422 (2014).

[62] C. C. Liu, H. Jiang, and Y. Yao, Phys. Rev. B 84, 195430 (2011).

[63] Z. Qiao, H. Jiang, X. Li, Y. Yao, and Q. Niu, Phys. Rev. B 85, 115439 (2012).

[64] V. E. Demidov, S. Urazhdin, H. Ulrichs, V. Tiberkevich, A. Slavin, D. Baither, G. Schmitz, and S. O. Demokritov, Nat. Mater. 11, 1028 (2012).

[65] A. Rycerz, J. Tworzyd, and C. W. J. Beenakker, Nat. Phys. 3, 172 (2007).

[66] H. Pan, Z. Li, C. C. Liu, G. Zhu, Z. Qiao, and Y. Yao, Phys. Rev. Lett. 112, 106802 (2014).

[67] L. E. Golub, S. A. Tarasenko, M. V. Entin, and L. I. Magarill, Phys. Rev. B 84, 195408 (2011).

[68] R. V. Gorbachev, J. C. W. Song, G. L. Yu, A. V. Kretinin, F. Withers, Y. Cao, A. Mishchenko, I.V. Grigorieva, K. S. Novoselov, L. S. Levitov, and A. K. Geim, Science 346, 448 (2014).

[69] D. A. Abanin, R. V. Gorbachev, K. S. Novoselov, A. K. Geim, and L. S. Levitov, Phys. Rev. Lett. 107, 096601 (2011).

[70] H. Min, G. Borghi, M. Polini, and A. H. MacDonald, Phys. Rev. B 77, 041407(R) (2008); J. E. Drut and T. A. Lahde, ibid. 79, 165425 (2009); Y. Araki, ibid. 84, 113402 (2011).

[71] B. Hunt, J. D. Sanchez-Yamagishi, A. F. Young, M. Yankowitz, B. J. LeRoy, K. Watanabe, T. Taniguchi, P. Moon, M. Koshino, P. Jarillo-Herrero, and R. C. Ashoori, Science 340, 1427 (2013).

[72] C. Ortiz Pauyac, X. Wang, M. Chshiev, and A. Manchon, Appl. Phys. Lett. 102, 252403 (2013).

[73] D. Xiao, W. Yao, and Q. Niu, Phys. Rev. Lett. 99, 236809 (2007); D. Xiao, M. Chang, and Q. Niu, Rev. Mod. Phys. 82, 1959 (2010).

[74] A. Sakai and H. Kohno, Phys. Rev. B 89, 165307 (2014).

[75] W. F. Tsai, C. Y. Huang, T. R. Chang, H. Lin, H. T. Jeng, and A. Bansil, Nat. Commun. 4, 1500 (2013).

[76] G. Giovannetti, P. A. Khomyakov, G. Brocks, P. J. Kelly, and J. van den Brink, Phys. Rev. B 76, 073103 (2007); X. Zhong, Y. K. Yap, R. Pandey, and S. P. Karna, ibid. 83, 193403 (2011); B. Sachs, T. O. Wehling, M. I. Katsnelson, and A. I. Lichtenstein, ibid. 84, 195414 (2011). 